\title{
Dynamical Model-Based Aerobics Upper Limb Inward and Outward Rotation Motion Sports Injured Part Research
}

\author{
Jianyang Wang ${ }^{1, *}$ and Jinchao Zhang ${ }^{2}$ \\ ${ }^{I}$ Wuhan Sports University, Wuhan, 430079, Hubei, China; \\ ${ }^{2}$ Institute of Physical Education, Central China Normal University, Wuhan, 430070, Hubei, China
}

\begin{abstract}
In the flourishing society, aerobics as a sport event that can build one's body and cultivate one's taste, is popular among ordinary people, but due to professional knowledge deficiency, mass may unconsciously generate injuries in body when taking aerobics exercise, in order to reduce occurrence rate of such injuries, the paper firstly gets dynamics relations and arms rotational inertia when aerobics athletes exercise upper limb inward and outward rotation motion by researching, gets that aerobics athletes' arms potential energy size when exercise upper limb inward and outward rotation motion, and according to momentum theory of transmitting from near-end to far-end, athletes can strengthen elbow joint momentum through shoulder joint effective braking effect, and then transfer momentum to wrist joint by elbow joint braking to have end acceleration effects. It also gets forearm angular speed should be bigger than that of upper arm so that can more beneficial to elbow joint acceleration. And it gets that when aerobics athletes take upper limb inward and outward rotation motion, due to it should let forearm angular speed to be bigger than upper arm's angular speed to drive elbow joint acceleration, athletes should let elbow joint speed to be more faster than shoulder joint in the process, and then shoulder joint is likely to suffer injuries because of braking, by comparing, elbow joint injury extent is far less than that of shoulder joint, athlete can take some corresponding prevention measures by referencing the result.
\end{abstract}

Keywords: Dynamics analysis, Rotational inertia, Moment equation, Dynamics transmitting, Sports injury.

\section{INTRODUCTION}

Since reform and opening-up, as economic strength constantly raised, people physical quality has also increasingly strengthened. Accompanying is arising of all kinds of sports events, while among these events, aerobics as a kind of sports events that can build one's body and also cultivate one's taste, it becomes favorite sport among masses, and also due to its good weight losing effects, is very popular among women [1]. Through aerobics, it not only can build healthy body but also can let people bodily form to become more and more symmetrical and perfect while take aerobics exercises [2].

With information globalization in eighties of 20th century, aerobics as an entertainment and fitness event has rapidly spread in the world and formed into a keep-fit fever. Fitness aerobics mainly spread in folk and is used for people physical exercises and mood cultivation [3]. Therefore, its motions are mostly simple and easier to learn, and rhythm is slower, which is suitable to group of people at all ages. Its main types can be divided into Latin bodybuilding exercise, street dancing, kickboxing, soft aerobics and aerobic gymnastics and so on.
Though types have differences, attributes are for improving personal physical quality, cultivating one's taste, perfecting bodily form and keeping psychological health and so on [4]. Aerobics require that motions should be standard and full of elasticity as well as better sense of rhythm. But if masses lack of standardization when exercise aerobics, then it is more possible to appear body partial injuries, and then the paper carries on analysis and researches on aerobics upper limb inward and outward rotation [5].

\section{MODEL ESTABLISHMENTS AND SOLUTION}

Aerobics basic motions is composed of head and neck, chest, waist, upper and lower limbs these five parts, basic forms are extending, flexing, cycling, turning, swinging, lifting and vibrating.Among them, it represents as wave motion, swinging, extension and flexion, turning, cycling, leapfrogging and dance steps and so on. Fig. (1) is aerobics upper limb inward and outward rotation motions' schematic Figure [6].

\subsection{Aerobics Upper Limb Inward and Outward Rotation Dynamics Analysis}

Rotational inertia refers to a rigid body generated inertia measurement when it makes fixed-axis rotation surrounding axis, it is only related to rigid body's shape, mass and axis location, and is uncorrelated to angular speed and other 


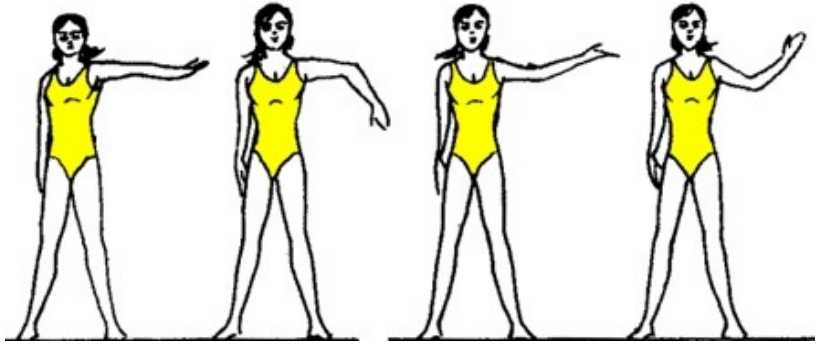

Fig. (1). Aerobics upper limb inward and outward rotation motions' schematic figure.

parameters. When aerobics athletes take upper limb inward and outward rotation motions, it can regard athlete arm as rigid body that makes fixed-axis rotation surrounding shoulder joint and elbow joint, Fig. (2) is athlete arm rotating schematic figure.
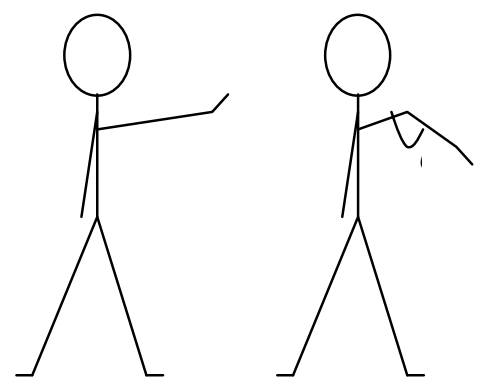

Fig. (2). Athlete arm rotation schematic figure.

Aerobics athlete generated rotational inertia is: $I=\Sigma M_{i} R_{i}^{2}$

Now regard aerobics athlete's body as mass continuous distribution rigid body, and can get:

$I=\iiint_{v} R^{2} d m=\iiint_{v} R^{2} \rho d V$

Among them, $M_{i}$ is human body each part mass, athlete each part to axis distance is $R, \rho$ is human body density. Aerobics athlete arm rotational tensor $\stackrel{\leftrightarrow}{Z}_{c}$ is:

$\stackrel{\leftrightarrow}{Z}_{c}=\iiint_{v} \rho\left(R^{2} \stackrel{\leftrightarrow}{E}-\vec{R} \vec{R}\right) d V$

In formula $\vec{R}=R_{1} \vec{e}_{1}+R_{2} \vec{e}_{2}+R_{3} \vec{e}_{3}$ is athlete body any point $O$ radius vector; two vectors' product is $\vec{R} \vec{R}$; Athlete body's unit tensor is: $\stackrel{\leftrightarrow}{E}=\left[\begin{array}{lll}1 & & \\ & 1 & \\ & & 1\end{array}\right]$

Therefore, athlete arm's unit orthogonal curvilinear frame is $\left(C ; \vec{e}_{1}, \vec{e}_{2}, \vec{e}_{3}\right)$.

Athlete upper limb inward and outward rotational tensor moment equation

When aerobics athlete takes upper limb inward and outward rotation, upper limb surrounding shoulder joint rotation's resultant moment vector is $\Sigma \vec{M}_{c}, \vec{\omega}$ is athlete inertia rotating system's angular speed vector, angular accelerated speed vector is $\vec{\gamma}$, therefore when aerobics athlete takes upper limb inward and outward rotation, the rotational tensor moment equation is

$\Sigma \vec{M}_{c}=\stackrel{\leftrightarrow}{Z}_{c} \bullet \vec{\gamma}+\vec{\omega} \times \stackrel{\leftrightarrow}{Z}_{c} \bullet \vec{\omega}$

Now project moment equation into three coordinate systems' coordinate axis, where can see athlete arm's moment equation in each coordinate axis direction.

When athlete takes inward and outward rotation, shoulder joint drives upper arm to rotate, it will produce a resultant moment $M_{1}$, formula is as following: $M_{1}=\varphi_{1} \bullet I_{1}$

Among them, upper arm rotational inertia is $I_{1}, \varphi_{1}$ is upper arm angular accelerated speed. And:

$I_{1}=\frac{M_{1} r_{1}^{2}}{2}$

$M_{1}$ is upper arm mass, $r_{1}$ is upper arm radius, upper arm angular accelerated speed $\varphi_{1}$ is:

$\varphi_{1}=\frac{d w_{1}}{d t}=\frac{d^{2} \theta_{1}}{d t^{2}}$

Then forearm final speed, except for itself will generate angular speed, it has an initial angular speed that is upper arm angular speed $\varphi_{1}$, so forearm angular speed $\varphi_{2}$ is:

$\varphi_{2}=\frac{d w_{2}}{d t}+\frac{d w_{1}}{d t}=\frac{d^{2} \theta_{2}}{d t^{2}}+\frac{d^{2} \theta_{1}}{d t^{2}}$

When aerobics athlete takes upper limb inward and outward rotation, arm generated gravity potential energy is: $G=m g\left(H_{1}-H_{2}\right)$

Now establish athlete's whole arm into three degree of freedom model that rotates around shoulder joint and elbow joint, as Fig. (3) show.

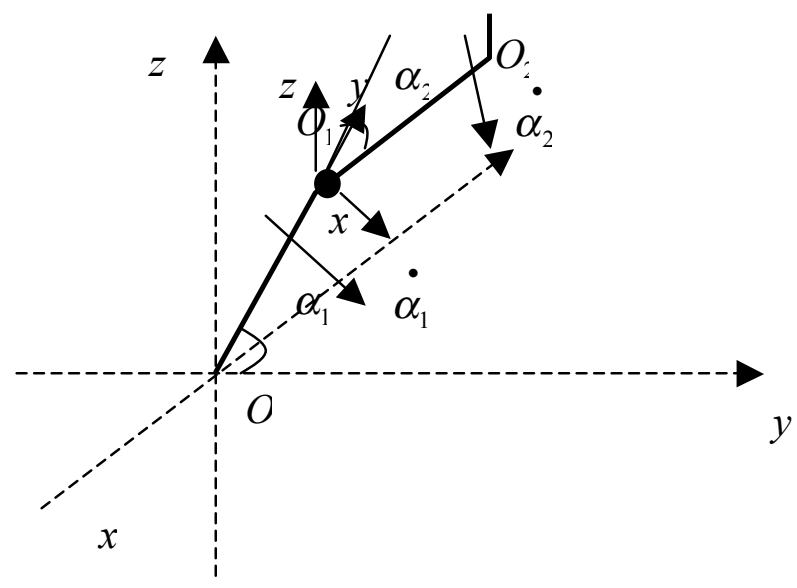

Fig. (3). Athlete arm rigid body rotation schematic figure. 
In Fig. (3), $O$ point is shoulder joint, $O_{1}$ is elbow joint, length of upper arm and forearm are respectively $l_{1}, l_{2}$, anatomic angles are respectively $\alpha_{1}, \alpha_{2}$. Among them, $O$ and $O_{1}$ are three degree of freedom, and trivector is $\dot{\alpha}_{1}, \dot{\alpha}_{2}$.

Now take earth as reference system, $\dot{\beta}_{1}$ and $\dot{\beta}_{2}$ are athlete upper arm and forearm's angular speed relative to reference system, elbow joint speed is $\dot{\alpha}_{2}$, its size is: $\dot{\alpha}_{2}=\dot{\beta}_{2}-\dot{\beta}_{1}$

By above formula, it can get when athlete takes upper limb inward and outward rotation motions, wrist angular speed is related to upper arm and forearm angular speeds and elbow joint angular speed. Due to upper arm and forearm are rotating, it can get elbow joint $O_{1}$ circular motion process, forearm makes rotation and translation in relative coordinate system $O_{1}-x y z$. And it is relative independent in rotation and translation that will not influence each other, so the two joints movement vectors sum is forearm angular speed. Then $\mathrm{O}_{2}$ point speed is related to $\mathrm{O}_{2}$ point speed relative to inertia rotation system and node $O_{1}$ linear speed relative to reference system, that:

$\vec{C}\left(\vec{O}_{1}\right)_{G}=\dot{\alpha}_{1} \times \vec{Y}_{1}=\dot{\beta}_{1} \times \vec{Y}_{1}$

$C\left(\vec{O}_{2}\right)_{L}=\dot{\alpha}_{2} \times \vec{Y}_{2}$

In formula $\vec{C}\left(\vec{O}_{1}\right)_{G}$ is elbow joint $O_{1}$ speed vector in reference system, $C\left(\vec{O}_{2}\right)_{L}$ is $O_{2}$ point speed relative to elbow joint $O_{1}$ point. $\dot{\beta}_{1}$ is upper arm rotational angular •

speed, $\alpha_{1}, \alpha_{2}$ are respectively shoulder joint $O$ and elbow joint $O_{1}$ angular speeds, $\vec{Y}_{1}$ is shoulder joint to elbow joint position vector, $\vec{Y}_{2}$ is elbow joint to wrist joint position vector.

Solve $O_{2}$ point relative to earth speed $\dot{O}_{2 G}=\vec{C}\left(\vec{O}_{2}\right)_{G}$, it should first solve upper arm and forearm partial movement influences on joints, and get according to vector theorem:

$\dot{O}_{2 G}=\dot{\alpha}_{1} \times \vec{Y}_{1}+\dot{\alpha}_{2} \times \vec{Y}_{2}+\dot{\alpha}_{1} \times \vec{Y}_{2}$

According to distribution rate, it gets:

$\dot{O}_{2 G}=\dot{\alpha}_{1}\left(\vec{Y}_{1}+\vec{Y}_{2}\right)+\dot{\alpha}_{2} \times \vec{Y}_{2}$

Simplify and get:

$\dot{O}_{2 G}=\vec{O}_{2 G} \times \dot{\alpha}_{1}+\dot{\alpha}_{2} \times \vec{Y}_{2}$
Among them, $\dot{O}_{2 G}$ is wrist joint position vector in reference system, cross product $\vec{O}_{2 G} \times \dot{\alpha}_{1}$ is wrist joint generated speed by shoulder joint action, and cross product $\dot{\alpha}_{2} \times \vec{Y}_{2}$ is wrist joint generated speed by elbow joint action.

In order to more precise describe each joint and ankle joint speed relations, now it can write Fig. (3) model shoulder, elbow joints' angles and wrist joint positions relations as:

$\left\{\begin{array}{l}x_{p}=Y_{1} \cos \alpha_{1}+Y_{2} \cos \left(\alpha_{1}+\alpha_{2}\right) \\ y_{p}=Y_{1} \sin \alpha_{1}+Y_{2} \sin \left(\alpha_{1}+\alpha_{2}\right) \\ z_{p}=Y_{1} \cos \alpha_{1}+Y_{2} \sin \left(\alpha_{1}+\alpha_{2}\right)\end{array}\right.$

Classify shoulder joint and elbow joint angles into infinitely small displacements, and relations with wrist joint positions vectors can get by above formula derivation:

$$
\left\{\begin{array}{c}
d X=\frac{\partial X\left(\alpha_{1}, \alpha_{2}\right)}{\partial \alpha_{1}} d \alpha_{1}+\frac{\partial X\left(\alpha_{1}, \alpha_{2}\right)}{\partial \alpha_{2}} d \alpha_{2} \\
d Y=\frac{\partial Y\left(\alpha_{1}, \alpha_{2}\right)}{\partial \alpha_{1}}+\frac{\partial Y\left(\alpha_{1}, \alpha_{2}\right)}{\partial \alpha_{2}} d \alpha_{2} \\
d Z=\frac{\partial Z\left(\alpha_{1}, \alpha_{2}\right)}{\partial \alpha_{1}}+\frac{\partial Z\left(\alpha_{1}, \alpha_{2}\right)}{\partial \alpha_{2}} d \alpha_{2}
\end{array}\right.
$$

Write above formula into matrix form as:

$$
\left(\begin{array}{l}
d X \\
d Y \\
d Z
\end{array}\right)=\left(\begin{array}{ll}
\frac{\partial X\left(\alpha_{1}, \alpha_{2}\right)}{\partial \alpha_{1}} & \frac{\partial X\left(\alpha_{1}, \alpha_{2}\right)}{\partial \alpha_{2}} \\
\frac{\partial Y\left(\alpha_{1}, \alpha_{2}\right)}{\partial \alpha_{1}} & \frac{\partial Y\left(\alpha_{1}, \alpha_{2}\right)}{\partial \alpha_{2}} \\
\frac{\partial Z\left(\alpha_{1}, \alpha_{2}\right)}{\partial \alpha_{1}} & \frac{\partial Z\left(\alpha_{1}, \alpha_{2}\right)}{\partial \alpha_{2}}
\end{array}\right)\left(\begin{array}{l}
d \alpha_{1} \\
d \alpha_{2}
\end{array}\right)
$$

By matrix attributes and vector product method, it can write above formula into: $d \vec{O}_{2}{ }_{G}=\vec{D} d \vec{\alpha}$, from which $\vec{D}=\left(\begin{array}{ll}\frac{\partial X}{\partial \alpha_{1}} & \frac{\partial X}{\partial \alpha_{2}} \\ \frac{\partial Y}{\partial \alpha_{1}} & \frac{\partial Y}{\partial \alpha_{2}} \\ \frac{\partial Z}{\partial \alpha_{1}} & \frac{\partial Z}{\partial \alpha_{2}}\end{array}\right)$

$\vec{D}$ is current structure infinitely small node angular displacement and wrist joint infinitely small displacement relations. Input matrix relations into above formula and can get: $\frac{d \vec{O}_{2 G}}{d t}=\vec{D} \frac{d \vec{\alpha}}{d t}$ or is $\vec{O}_{2 G}=\vec{D}\left[\dot{\alpha}_{1}, \dot{\alpha}_{2}\right]^{T}$, input it into wrist joint relative speed computational formula and can get:

$$
\dot{O}_{2 G}=\left(\begin{array}{ll}
\frac{\partial X}{\partial \alpha_{1}} & \frac{\partial X}{\partial \alpha_{2}} \\
\frac{\partial Y}{\partial \alpha_{1}} & \frac{\partial Y}{\partial \alpha_{2}} \\
\frac{\partial Z}{\partial \alpha_{1}} & \frac{\partial Z}{\partial \alpha_{2}}
\end{array}\right)\left[\vec{\alpha}_{1}, \vec{\alpha}_{2}\right]^{T} \dot{\alpha}_{1}+\dot{\alpha}_{2} \times \vec{Y}_{2}
$$


After that, apply Lagrange equation to establish restrained particle dynamical equation; define Lagrange function $L$ as system kinetic energy $K$ and position energy $P$ difference: $L=K-P$

System dynamical equation is:

$$
F_{i}=\frac{d}{d t}\left(\frac{\partial L}{\partial \dot{q}_{i}}-\frac{\partial L}{\partial q_{i}}\right) \quad i=1,2, \cdots, n
$$

In above formula, $\dot{q}_{i}$ is corresponding speed, $q_{i}$ is kinetic energy and position energy coordinate, $F_{i}$ represents the $i$ coordinate acting force, upper arm and forearm as well as coordinate axis included angles are respectively $\alpha_{1}, \alpha_{2}$, lengths are respectively $l_{1}, l_{2}$, distance between upper arm and forearm mass center and shoulder and elbow joints are respectively $j_{1}, j_{2}$, therefore, it is clear that upper arm mass center coordinate $\left(X_{1}, Y_{1}\right)$ is:

$$
\left\{\begin{array}{l}
X_{1}=j_{1} \sin \alpha_{1} \quad Y_{1}=j_{1} \cos \alpha_{1} \\
X_{2}=l_{1} \sin \alpha_{1}+j_{2} \sin \left(\alpha_{1}+\alpha_{2}\right) \quad Y_{2}=-l_{1} \cos \alpha_{1}-j_{2} \cos \left(\alpha_{1}+\alpha_{2}\right)
\end{array}\right.
$$

Similarly, forearm mass center coordinate $\left(X_{2}, Y_{2}\right)$ can also be solved. System kinetic energy $E_{k}$ and system potential energy $E_{p}$ expression is:

$$
\left\{\begin{array}{l}
E_{k}=E_{k 1}+E_{k 2}, E_{k 1}=\frac{1}{2} m_{1} j_{1}^{2} \dot{\alpha}_{1}^{2} \\
E_{k 2}=\frac{1}{2} m_{2} l_{1}^{2} \dot{\alpha}_{1}^{2}+\frac{1}{2} m_{2} \dot{j}_{2}^{2}\left(\dot{\alpha}_{1}+\dot{\alpha}_{2}\right)^{2}+m_{2} l_{2} j_{2}\left(\dot{\alpha}_{01}^{2}+\dot{\alpha}_{1} \dot{\alpha}_{2}\right) \cos \dot{\alpha}_{2} \\
E_{p}=E_{p 1}+E_{p 2}, E_{p 1}=\frac{1}{2} m_{1} g j_{1}\left(1-\cos \alpha_{1}\right) \\
E_{p 2}=m_{2} g j_{2}\left[1-\cos \left(\alpha_{1}+\alpha_{2}\right)\right]+m_{2} g l_{1}\left(1-\cos \alpha_{1}\right)
\end{array}\right.
$$

Write above formula into Lagrange function expression, by Lagrange system dynamics equation, it can get shoulder joint and wrist joint momentum $M_{j}$ and $M_{z}$ are:

$$
\left[\begin{array}{l}
M_{j} \\
M_{z}
\end{array}\right]=\left[\begin{array}{ll}
D_{11} & D_{12} \\
D_{21} & D_{22}
\end{array}\right]\left[\begin{array}{l}
\ddot{\alpha_{1}} \\
\ddot{\alpha_{2}}
\end{array}\right]+\left[\begin{array}{ll}
D_{111} & D_{122} \\
D_{211} & D_{222}
\end{array}\right]\left[\begin{array}{c}
\dot{\alpha}_{1} \\
\dot{\alpha}_{1} \\
\alpha_{2}
\end{array}\right]+\left[\begin{array}{cc}
D_{112} & D_{121} \\
D_{212} & D_{221}
\end{array}\right]\left[\begin{array}{c}
\dot{\alpha_{1}} \dot{\alpha_{2}} \\
\dot{\alpha_{2}} \dot{\alpha}_{1}
\end{array}\right]+\left[\begin{array}{l}
D_{1} \\
D_{2}
\end{array}\right]
$$

In above formula, $D_{i j k}$ is:

$$
\begin{aligned}
& {\left[\begin{array}{llll}
D_{111} & =0 & D_{222}=0 & D_{121}=0
\end{array} \quad D_{22}=m_{2} j_{2}^{2}\right.} \\
& D_{11}=m_{1} j_{1}^{2}+m_{2} j_{2}^{2}+m_{2} l_{1}^{2}+2 m_{2} l_{1} j_{2} \cos \alpha_{2} \\
& D_{1}=\left(m_{1} j_{1}+m_{2} l_{1}\right) g \sin \alpha_{1}+m_{2} j_{2} g \sin \left(\alpha_{1}+\alpha_{2}\right) \\
& D_{12}=m_{2} j_{2}^{2}+m_{2} l_{1} j_{2} \cos \alpha_{2} \quad D_{21}=m_{2} j_{2}^{2}+m_{1} l_{1} j_{2} \cos \alpha_{2} \\
& D_{122}=-m_{2} l_{1} j_{2} \sin \alpha_{2} \quad D_{211}=m_{2} l_{1} j_{2} \sin \alpha_{2} \\
& {\left[D_{112}=-2 m_{2} l_{1} j_{2} \sin \alpha_{2} \quad D_{212}=D_{122}+D_{211} \quad D_{2}=m_{2} j_{2} g \sin \left(\alpha_{1}+\alpha_{2}\right)\right]}
\end{aligned}
$$

By above formula analysis, it can get when aerobics athlete takes upper limb inward and outward rotation motions, he should try to speed up arm speed so that can let
$O_{2 G}$ vertical direction projection speed to be accelerated to maximum value, and according to matrix vector theorem, it is clear only when $\alpha_{1}, \alpha_{2}$ meet constraint condition $45^{\circ}<\alpha^{1}+\alpha^{2}<90^{\circ}, \quad 0<\dot{\alpha}_{1}<\dot{\alpha}_{2}$, wrist joint speed can get maximum value in sagittal plane. That let athlete upper limb inward and outward rotation movement preliminary phase upper arm and forearm descending flex should try not to surpass $45^{\circ}$, and its anatomic angle changing speed should be fast so that can arrive at muscle mechanics muscle relaxation feature. And because when $\alpha_{1}, \alpha_{2}$ meet its constraint conditions, $\dot{\alpha}_{1}$ and $\dot{\alpha}_{2}$ increase, when aerobics athlete takes upper limb inward and outward rotation movement, it should let athlete upper arm and forearm anatomic angle change rate arrive at maximum value in unit time, and also require athlete forearm anatomic angle change rate to be bigger than upper arm angle change rate during the process. By rigid body transferability, it can know that when arm's upper arm and forearm are nearly in $180^{\circ}$, force transfers to forearm along upper arm axis, but due to upper arm and forearm are connected, then it will lead to force attenuation, therefore forearm angular speed bigger than upper arm angular speed is more beneficial to elbow joint acceleration.

By above analysis, it can get when aerobics athlete takes upper limb inward and outward rotation movement, due to it should let forearm angular speed to be bigger than upper arm angular speed to drive elbow joint acceleration, athletes should let elbow joint speed to be more faster than shoulder joint in the process, and then shoulder joint is likely to suffer injuries because of braking, by comparing, elbow joint injury extent is far less than that of shoulder joint.

\section{CONCLUSION}

By researching, the paper gets dynamics relations and arms rotational inertia when aerobics athlete exercises upper limb inward and outward rotation motion, and aerobics athletes' arms potential energy size when aerobics athlete exercises upper limb inward and outward rotation motion, besides according to momentum theory of transmitting from near-end to far-end, athletes can strengthen elbow joint momentum through shoulder joint effective braking effect, and then transfer momentum to wrist joint by elbow joint braking to have end acceleration effects. It also gets forearm angular speed should be bigger than that of upper arm so that can more beneficial to elbow joint acceleration. And it gets that when aerobics athlete takes upper limb inward and outward rotation motion, due to it should let forearm angular speed to be bigger than upper arm's angular speed to drive elbow joint acceleration, athletes let elbow joint speed to be more faster than shoulder joint in the process, and then shoulder joint is likely to suffer injuries because of braking, by comparing, elbow joint injury extent is far less than that of shoulder joint.

\section{CONFLICT OF INTEREST}

The authors confirm that this article content has no conflict of interest. 


\section{ACKNOWLEDGEMENTS}

None declared.

\section{REFERENCES}

[1] F. Chen, "Sports injuries occurred to aerobic exercises and its prevention methods," High Education Forum, no. 6, 2004.

[2] J. Kou, "Dancing injuries occurrence and prevention," Journal of Qinghai University, vol. 22, no. 4, pp. 103-104, 2006.
[3] X. Li, "Discussion on injuries and prevention in aerobics," China Sports Medicine Magazine, vol. 21, no. 4, pp. 424-425, 2002.

[4] Y. Li, Sports Training Biomechanics Foundation, Science and Technology Press, Wuhan Hubei, 2003, vol. 10.

[5] Y. Lu, and H. Li, "Universities sports major aerobics special elective students' sports injuries causes' analysis," Notification of Sports Scientific and Technical Literature, no. 9, 2009.

[6] X. Long, "Universities aerobics sport injuries causes' analysis," Sports Science and Technology, no. 2, 2007.

Received: June 10, 2015

Revised: July 29, 2015

Accepted: August 15, 2015

(C) Wang and Zhang; Licensee Bentham Open.

This is an open access article licensed under the terms of the (https://creativecommons.org/licenses/by/4.0/legalcode), which permits unrestricted, noncommercial use, distribution and reproduction in any medium, provided the work is properly cited. 